\title{
Application of the damage index method for plate-like structures to timber bridges
}

\author{
B. Samali ${ }^{*} \dagger$, J. Li, F. C. Choi and K. Crews \\ Centre for Built Infrastructure Research (CBIR), Department of Civil and Environmental \\ Engineering, Faculty of Engineering and IT, University of Technology Sydney, PO Box 123, \\ Broadway, NSW 2007, Australia
}

SUMMARY

The paper presents a research recently completed by the authors utilising a method of damage evaluation for identifying damage in timber bridges, numerically and experimentally. The method utilises changes in modal strain energy between the undamaged and damaged states of plate-like structures. A finite element model of a laboratory timber bridge was developed to investigate the capabilities and limitations of the method to detect damage. A simple four-girder bridge was fabricated and tested in a laboratory to verify the method. The numerical studies showed that the method can correctly identify single and multiple damage locations within the bridge. The experimental studies also showed promising results for detecting severe damage, but less effective for light and medium damage.

KEY WORDS: damage detection; timber bridge; damage index method; plate-like structures

\section{INTRODUCTION}

Timber bridges have contributed significantly to the development of many countries until they were slowly replaced by iron, steel and concrete in early $20^{\text {th }}$ century. Besides serving as means for transportation, timber bridges, nowadays also have significant heritage values. According to the Department of Transport and Regional Services Australia (DOTARS) [1], there are approximately 29,000 timber bridges in Australia, many are still in service. The enormous number of timber bridges, which form a significant portion of bridge assets, reflects the importance of these structures to the country's economic growth and the daily life of Australians.

In light of the long history of timber bridges in Australia, DOTARS [1] has estimated that a third of them are in excess of 50 years old and some of these bridges are functionally obsolete and are structurally deficient. Due to their age and ill 'health' condition, it is necessary to perform periodic inspections on them to ensure the safety of these structures as well as to preserve these heritage-valued structures of the country [2].

*Correspondence to: B. Samali, Department of Civil and Environmental Engineering, Faculty of Engineering \& IT, Building 2, Level 5, University of Technology Sydney, PO Box 123, Broadway, NSW 2007, Australia.

†E-mail: Bijan.Samali@uts.edu.au

Tel: +61-2-9514 2023

Fax: +61-29514 2868 
Often the problems in wood/timber structures lie not in the effectiveness of preserving systems of the material itself but in not having the correct tools necessary to locate deterioration [3]. Thereby, it delays application of remedial treatments to prevent further degradation in the structures. The current-state-of-the-practice for condition assessment of timber bridges is through destructive and semidestructive means. A wide variety of techniques are being used to verify deterioration or rot in timber members [4]. Resistance drilling, probing and coring are all destructive or semidestructive methods that are utilised to assess deterioration or rot in in-service wood members.

Visual inspection, acoustics and stress wave or ultrasonic based techniques are common non-destructive techniques (NDTs) used either individually or in combination to evaluate woodbased materials. However, these methods are mostly used on small and accessible areas. It is time consuming to perform them on large timber structures like timber bridges and it is also costly. This led to the need for global methods that would be able to reduce testing time, the cost involved and disruption to traffic flow. The global methods, if necessary, can then combine with other more localised non-destructive evaluation (NDE) methods for evaluating damage details.

Over the last few decades, many methods of structural integrity assessment for timber bridges have been developed. The development in research for in-situ NDE and NDT for decay detection and subsequent strength assessment are highlighted as one of the needs and goals for research in a workshop published by ASCE [5]. Preceding to the ASCE workshop Ou and Weller [6] also reported concerns on overlooked studies in inspection of timber bridges in early eighties. Proof load testing is one of the commonly used methods, but it has a number of deficiencies, including endangering the remaining strength of a tested bridge. Therefore, recently more work has been carried out to assess the in-service stiffness of timber structures like short-span timber bridges and floor systems within buildings using vibration techniques. A simple dynamic testing method has been developed by Li et al. [7] to evaluate the overall stiffness and strength of inservice timber bridges. This dynamic method is generally proven to be easily performed and cost effective. In addition, the operators are not required to have high skills and can be easily trained on job to perform the dynamic timber testing. Application of vibration techniques for condition assessments in timber bridges were also found in works by Peterson et al. [8-9], Peterson et al. [10], Yang et al. [11], Morison et al. [12-13], Hu at al. [14-15], Hu and Afzal [16-17], and Ross et al. [18].

Development of NDE or NDT is generally divided into two major groups, that is, localised methods and global methods. In Australia, for localised methods, nuclear densometer method and resistograph method have been used. The nuclear densometer is a real time density measuring system that produces a graph showing the net thickness of the timber section [19]. The work shows that change in thickness due to deterioration can be measured within the tested sections. Resistograph or resistance drilling method is a quasi static method that measures the resistance of the timber to the penetration of a small $1.4 \mathrm{~mm}$ diameter drill bit. The method shows that with deterioration, the drill will penetrate deeper into the girder. The quantification of the damage is possible [19-20]. For the global methods, as mentioned above, Li et al. [7] have developed a dynamic testing method to evaluate the global structural stiffness and strength of timber bridges. The authors are progressively expanding the research work to detect the location of damage and evaluate damage severity.

This paper reports on a project recently completed by the authors to develop and implement dynamic methods for evaluation of damage in timber bridges. The damage index method for plate-like structures (DI-P) was adopted for damage detection, where the method 
involved the use of modal strain energy. The project involved numerical studies of a laboratory timber bridge subjected to single and multiple damage scenarios. The method was then verified by a scale timber bridge constructed under laboratory conditions. Experimental modal analysis was employed to obtain the modal parameters to be used in the DI-P method.

\section{FINITE ELEMENT MODEL}

\subsection{Undamaged model}

The finite element (FE) model of a laboratory timber bridge, scaled to maintain dynamic similitude representing real timber bridges in terms of their first frequencies, which usually range from $5 \mathrm{~Hz}$ to $20 \mathrm{~Hz}$, was constructed using a commercial FE software package, namely, ANSYS [21]. The basic dimensions of the bridge model are illustrated in Figure 1. The specimen's breath was $2,400 \mathrm{~mm}$ with a span length of $4,500 \mathrm{~mm}$. The four girders $(45 \mathrm{~mm}$ wide $\mathrm{x} 90 \mathrm{~mm}$ deep) and the deck $(21 \mathrm{~mm}$ thick) were modelled based on radiata pine timbers and structural plywood of grade F11, respectively, with modulus of elasticity obtained from four point bending tests. The solid elements (SOLID45) were utilised to model the girders, while shell elements (SHELL63) were used to model the deck. The screw connections between the deck and girders were modelled using rigid link (MATRIX27 hereafter referred as screw link) to transfer the rotational degrees of freedom (DOFs) from shell to solid elements as illustrated in Figure 2. The stiffness of the screw-link elements controls the level of composite action between the deck and girders. All the test results for timber beams and plywood, as well as the strength of the screw connections, were inputs to the model. The material properties for the deck were considered orthotropic, whilst those of the girders were isotropic. For the supports, pin-pin boundary conditions were used with additional link elements (MATRIX27, referred as support-links) to simulate the real boundary condition as shown in Figures 3 and 4. These were considered appropriate since the girders were actually $100 \mathrm{~mm}$ above the pivot point of the fabricated pins as depicted in Figure 4. Degrees of freedom (DOFs) were constrained at the contact points between support-links and girders. Meanwhile, both translational and rotational DOFs were constrained for the contact points between support-links and rigid frame, in the three directions corresponding to $\mathrm{X}, \mathrm{Y}$ and $\mathrm{Z}$ axes. In the bridge model, the stiffness of the support-links was adjusted manually during the calibration using natural frequencies as the response feedback. Mode shapes of 81 coordinates were extracted from the bridge model. The "as is" mode shapes were then reconstructed to be 1684-point mode shapes using bi-cubic spline interpolation technique. The reconstructed mode shapes with finer coordinates usually produce better damage detection results [22].

\subsection{Damaged model}

Twelve damage cases of the laboratory timber bridge were created in this research project using the FE model and they correspond to the experimental studies. The damage cases reported in this

paper are listed in Table 1 consisting of rectangular openings from the soffit of the beam. The damage to be simulated is pockets of rot or termites attack, typically found in timber bridges. The damage was inflicted at different girders and locations along the girder, such as $2 / 8$, midspan (4/8), 5/8 and 6/8 of the span length, to simulate rot in wood. The damage inflicted in the model is that of single and multiple damage scenarios, which correspond to the damage scenarios in experimental tests. The damage scenarios are featured in Table 1 and Figure 5. The ' $L$ ', ' $M$ ' and ' $\mathrm{S}$ ' damage denote levels of damage severity for each damage location, that is, 'light', 'medium' 
and 'severe' corresponding to $27.1 \%, 65.7 \%$ and $87.5 \%$ loss of 'I' (moment of inertia), respectively. The nine mode shapes extracted from the FE model are shown in Figure 6. In this paper, a few selected damage cases (as highlighted in Table 1) are presented to demonstrate the results of damage detection using the modal based damage detection method.

\section{EXPERIMENTS}

\subsection{Four-girder laboratory timber bridge}

A four-girder laboratory timber bridge was built in the laboratory to verify the damage detection method for timber structures. The basic dimensions of the structure are shown in Figure 1. The bridge consisted of four girders of treated radiata pine sawn timber measuring $45 \mathrm{~mm} \times 90 \mathrm{~mm}$ in cross section with a span length of $4.5 \mathrm{~m}$. The deck consisted of four pieces of $21 \mathrm{~mm}$ thick $\mathrm{x}$ $2.4 \mathrm{~m}$ wide and $1.2 \mathrm{~m}$ long structural plywood of grade F11. The deck and girders were connected using 50mm self-tapping screws with $137.5 \mathrm{~mm}$ spacing. No gluing was applied in order to avoid fully-composite action, to simulate the interactions in a real timber bridge. The longitudinal modulus of elasticity (MOE) was determined using four-point bending test for each of the four girders and the deck. This was done in order to select timbers that have similar material properties and used for material property input in the finite element models. The moisture content was estimated to be around $7-8 \%$ for the beam and was around $11-12 \%$ for the plywood using a wood moisture detector. The ends of the bridge girders were supported on concrete blocks, and rigidly connected to the strong floor. A specially designed support system as shown in Figure 4 was used between girders and the concrete block to ensure a well-defined boundary condition that is very close to a pin-pin condition. The full bridge model is depicted in Figure 7. The laboratory timber bridge, namely, Deck2, was used to designate the undamaged state as denoted by D2. The results form the basis of comparison for various damage cases utilising the extracted modal parameters and their derivatives.

\subsection{Inflicted damage in laboratory timber bridge}

The inflicted damage cases considered for the experimental studies are described in Table 1 and Figure 8. The three inflicted damage severities are shown in Figure 9. In this paper, similar damage cases correspond to the numerical studies in order to demonstrate and correlate the damage detection results.

\subsection{Experimental modal analysis}

The modal test set up and its instrumentation layout is shown in Figure 10. The processing of results is based on the use of digital signal processing techniques and using the fast Fourier transform (FFT) algorithm to process the time domain data to produce frequency domain data in terms of frequency response function (FRF) between different points on the test structure. In this study, the modal testing and experimental modal analysis yield natural frequency, damping ratio and their corresponding mode shapes. The tests were mainly performed under similar environmental conditions, that is, temperature of $22^{\circ} \mathrm{C} \pm 2^{\circ}$ and ambient humidity of $60 \% \pm 5 \%$ to minimise environmental factors on the modal parameters.

The system identification method used in this study employs an impact modal hammer to excite the test sample at the reference or driving point as shown in Figure 8. The impact location (reference or driving point), at 3/4 of the span length and directly located above girder 2 (g2), is a strategic location and can excite more modes simultaneously. The response signal along the span 
was recorded using nine low impedance piezoelectric accelerometers and one stationary accelerometer was used at the driving point. For the laboratory timber bridge, there were 81 measuring points as depicted in Figure 8. Due to limited number of sensors available, the data acquisition for the 81 points was done in 9 tests. The nine non-stationary accelerometers were moved from line-to-line, i.e., from line 1 (L1) to line 9 (L9) as illustrated in Fig. 8, until all measurement locations were covered. These accelerometers were used to measure the acceleration response on the top surface of the laboratory timber bridge as illustrated in Figure 10. The nine measurement points per line were deemed sufficient for accurate reconstruction of mode shapes using interpolation techniques. The driving point measurement (3/4 of span length on L4 or girder 2 (g2), refer to Figure 8 ) enabled the experimental mode shapes to be mass normalised. Each accelerometer with a magnetic base was attached onto a small steel plate, with approximate dimensions of $40 \mathrm{~mm} \times 90 \mathrm{mmx} 1 \mathrm{~mm}$, and secured on the top of the deck. The distance between each accelerometer was $1 / 8$ of the span length $(562.5 \mathrm{~mm})$ starting from one end support of the bridge to the other end as shown in Figures 8 and 10.

The frequency response functions (FRFs) were acquired using LMS CADA-X [23] signal acquisition module and a dynamic analyser HP E1432A Vxi. A typical acquired FRF spectrum using the system for the laboratory timber bridge is shown in Figure 11. The measurements were recorded using the dynamic response at $10,000 \mathrm{~Hz}$ sampling rate for a frequency span of $500 \mathrm{~Hz}$ and 8,192 data points, thus giving a resolution of $0.061 \mathrm{~Hz}$ per data point. By employing an averaging of 5 ensembles, this reduces the interference of noise.

Using the modal analysis module in the LMS CADA-X [23] software, a "frequency domain direct measurement" curve-fitting technique was used to extract the natural frequencies, damping ratios and mode shapes from the measured FRF data. From the modal testing and experimental modal analysis, nine vibration modes ranging from $8 \mathrm{~Hz}$ to $60 \mathrm{~Hz}$, were captured. The captured mode shapes are shown in Figure 12. From the 9x9-point experimental mode shapes, the 41x41-point mode shapes were reconstructed using two-dimensional (2-D) cubic spline interpolation technique, generating mode shape vectors with 41 coordinates in both longitudinal and transverse directions. The reconstructed mode shapes with finer coordinates have better chance of locating damage compared to using coarse coordinates. The reconstructed mode shapes were then applied in the damage detection algorithms attempting to locate and evaluate the inflicted damage scenarios. It was noted that the first and second torsional modes captured (modes 2 and 5) were not very good because the impact location was close to their node points. Nevertheless, these modes are still used in the subsequent discussions.

\section{DAMAGE DETECTION}

\subsection{Damage index for plate-like structures}

In research presented by Cornwell et al. [24], it was stated that using the damage index (DI) method developed for beam-like structures on a plate-like structure, by slicing the plate into beam stripes, was not expected to perform best in damage detection in comparison with the use of damage index method for plate-like structures (utilising two-dimensional curvatures). It is because the DI method for beam-like structures does not preserve torsional stiffness between slices. Nevertheless, it should be noted that the one-dimensional damage index method has been applied to two-dimensional and three-dimensional structures, such as a timber bridge [10], but by slicing them into beam-like elements. In this investigation, for the complicated laboratory timber bridge (a deck-girder system), the proposed damage index algorithm for plate-like structures 
(abbreviated as DI-P) by Cornwell et al. [24] was adopted to locate damage. The application of DI-P method to detect damage in timber structures has not been reported to the best knowledge of the authors.

Cornwell et al. [24] extended the damage index method for beam-like structures to include the detection of damage for plate-like structures characterised by a two-dimensional (2D) mode shape slope and curvature. The algorithm used to calculate the damage index for the $j k$ th subregion and the $i$ th mode, $\beta_{i j k}$, is given below for detecting location of damage.

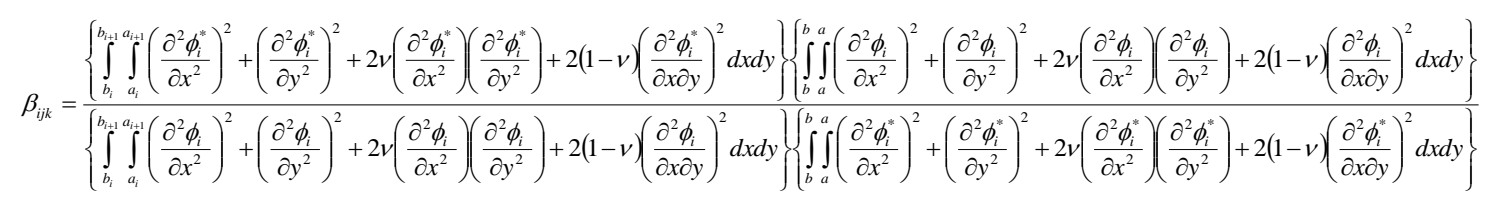

where $v$ represents Poisson's ratio and the term $\partial^{2} \phi_{l} / \partial x^{2}$ is a vector of second derivatives of mode shape coordinates (curvatures) with respect to $x$-axis. Similar convention is applicable to second derivatives with respect to $y$-axis and cross derivatives with respect to $x$ - and $y$-axes. Equation (1) denotes damage index in matrix form describing change of strain energy before and after damage, corresponding to mode $i$ for a bridge structure. The asterisk denotes the damage cases. To account for all available modes, $N M$, the damage indicator value for a single subregion $j k$ is given as:

$$
\beta_{j k}=\frac{\sum_{i=1}^{N M} N_{i j k}}{\sum_{i=1}^{N M} \operatorname{Denom~}_{i j k}}
$$

where $N u m_{i j k}=$ numerator of $\beta_{i j k}$ and Denom $_{i j k}=$ denominator of $\beta_{i j k}$ in (1), respectively. Transforming the damage indicator values into the standard normal space, normalised damage index $Z_{j k}$ is obtained:

$$
Z_{j k}=\frac{\beta_{j k}-\mu_{\beta_{j k}}}{\sigma_{\beta_{j k}}}
$$

where $\mu_{\beta j k}=$ mean of $\beta_{j k}$ values for all subregions $j k$ and $\sigma_{\beta j k}=$ standard deviation of $\beta_{j k}$ for all subregions $j k$. A judgment based threshold value is selected and used to determine which of the subregions $j k$ are possibly damaged which in real applications is left to the user to define based on what level of confidence is required for localisation of damage within the structure.

\section{RESULTS AND DISCUSSIONS}

In the following results, the normalised damage indicator, $Z_{j k}$, for each of the damage cases is plotted against the laboratory timber bridge span length $(4.5 \mathrm{~m})$ and width $(2.4 \mathrm{~m})$. In principle, when the statistically normalised damage indicator value of $Z_{j k}$ for a given location is larger than or equals to two (the probability-based criterion for damage), it is considered that damage existed at that location. The localisation of damage using the criterion, $Z_{j k} \geq 2$, was adopted as it would 
provide an approximately 95\% level of confidence in indicating the actual damage locations $[25,8]$.

To show the damage site in the graphs, the actual damage locations are marked by an arrow sign and labelled with 'Damage' in all subsequent figures. For damage localisation of the laboratory timber bridge, two types of graphs were used selectively in this study, that is, surface and contour plots. The surface plots provide an indication of the distribution of the damage indicators that are above zero $\left(Z_{j k}>0\right)$, while the contour plots give information on predicted damage locations with $Z_{j k} \geq 2$.

\subsection{Effects of sensors density}

The density of sensors (e.g. accelerometers) is an important factor in producing reliable damage detection results using vibration based methods. The selection of number and location of sensors in a structure have a major influence on the quality of the damage detection [26-27]. It is commonly acknowledged that as more information is obtained, the damage detection will be more accurate. When the collected data is below a certain level, the results of damage detection inevitably become unreliable. This implies that a large number of sensors are preferred in a modal test in order to produce accurate damage detection results. However, it is often found that it is impractical and very costly to have a dense sensor array. This is unlike cases in the finite element modelling, where the density is only associated with the mesh size and can be easily changed. In the experimental work, a reasonable number of sensors are essential to produce optimal damage detection results. In this investigation, 9x9 equally spaced sensors were used to acquire the responses of the test sample in the modal tests. With the two-dimensional (2-D) cubic spline interpolation technique, the limited data was expanded to forty one data points in both longitudinal and transverse directions of the span, which would enhance the effectiveness of the damage detection technique.

The comparison of damage localisation results obtained from the 'as is' experimental data and the interpolated experimental data using 2-D cubic spline were made using the DI-P method with the first nine modes on a three damage location case. From the contour plot of Figure 13(a), using the 'as is' data for the calculation of damage indicator with the first nine modes, we failed to locate the damage at position $(2.25 \mathrm{~m}, 2.1 \mathrm{~m})$, which is $2.25 \mathrm{~m}$ along the span length and $2.1 \mathrm{~m}$ across the width (the convention of $(2.25 \mathrm{~m}, 2.1 \mathrm{~m}$ ) is used in the following discussions) for case g2Sg4Sg3S. Even though using the 'as is' data in the DI-P method was able to detect two out of three severe damage locations, it is considered insufficient as it would potentially pose a danger to a structure if any severe damage remains detected. A 2-D cubic spline interpolation was adopted for the plate-like structure to expand the number of data points from the 'as is' data in order to enhance the possibility of damage detection using the DI-P method [28]. In the contour plot of Figure 13(b), applying the expanded data using the 2-D cubic spline technique, the method has clearly shown the locations of damage with two false positives (indication of spurious damage locations). The one missed damage location by using the 'as is' data may have been caused by the estimation of discrete curvature at the midspan $(2.25 \mathrm{~m})$ being overshadowed by the two adjacent equal or higher severity damage at $1 / 4$ and $3 / 4$ span $(1.125 \mathrm{~m}$ and $3.375 \mathrm{~m}$ ). Adding more data points between the original data points subsequent to interpolation, the difference of curvature between two adjacent damage locations was better defined. Hence, from the reconstructed data, the damage at midspan was not subsided. However, it is important to note that there is slight distortion of data near both supports as depicted in the contour plot of Figure 13(b), which are not seen in Figure 13(a). This may be due to the cubic nature of the 
interpolation and may be avoided by using a high-order interpolation technique as suggested by Worden et al. [29]. It is anticipated that such data distortion may also happen to the free edges. (Improving the cubic spline mode shape reconstruction technique to avoid the distortion of data was not included in the scope of work for this study and it is proposed to be undertaken in future work.) In general, it is obvious that without sufficient number of sensors, in many circumstances the damage localisation method cannot produce reliable results.

\subsection{Effects of number of modes used}

The numerical data of a laboratory timber bridge developed using a finite element model was used to study the effect of number of modes used in the DI-P method. As the number of modes captured, experimentally, is limited to nine, the first three and first nine modes were used, respectively, to compare to their performance in detecting damage. The first three modes are considered as these modes are easily acquired for a real timber bridge and the combination of modes is also commonly used [10]. However, the first three modes may not be sufficient to identify all damage locations. Hence, it is suggested to adopt all the acquired modes ( 9 modes) in the damage localisation algorithm [24]. In addition, with the latest developments in sensor technologies, it is possible nowadays to obtain the higher modes in a real structure.

To study the effect of number of modes used, a three damage location case of numerical results was used. The results of the damage indicator using the DI-P method are shown in Figure 14. Using the first three modes in the method for damage cases g2Sg4Sg3S (see the contour plot of Figure 14(a)), the method could not detect the severe damage at positions $(3.375 \mathrm{~m}, 0.9 \mathrm{~m})$ and $(1.125 \mathrm{~m}, 1.5 \mathrm{~m})$. While for the same case using nine modes, as illustrated in the contour plot of Fig.14b, all severe damage locations were identified. It is noted also that the damage index values for the three severe damage locations, calculated with nine modes (see surface plot of Figure 14(b)), are quite similar and agreed well with the actual damage condition. This shows that using a higher number of modes improves damage detection using the DI-P method.

\subsection{Damage localisation}

Based on the findings above, the following damage localisation results for the laboratory timber bridge were calculated using the first nine modes in the damage index method for plate-like structures (DI-P).

\subsubsection{Numerical results}

For damage localisation using the DI-P method in the numerical studies, the paper presents a few damage cases of single and two damage locations. The single damage case applied the DI-P method and computed, using the first nine modes and the numerical data. The results are illustrated in Figure 15. For single damage cases of light, medium and severe (g2L, g2M and $\mathrm{g} 2 \mathrm{~S})$, the DI-P method was able to identify the damage location at position $(3.375 \mathrm{~m}, 0.9 \mathrm{~m})$ as shown in the contour plots of Figures 15(a) to (c).

The results of two damage cases using the DI-P method are shown in Figure 16. It is obvious that all severe damage locations at position $(3.375 \mathrm{~m}, 0.9 \mathrm{~m})$ were identified for damage cases g2Sg4L, g2Sg4M and g2Sg4S as shown in Figures 16(a) to (c), respectively. The medium damage at position $(2.25 \mathrm{~m}, 2.1 \mathrm{~m})$ was also identified for case g2Sg4M (see Figure 16(b)). However, the light damage at position $(2.25 \mathrm{~m}, 2.1 \mathrm{~m})$ was not identifiable for case $\mathrm{g} 2 \mathrm{Sg} 4 \mathrm{~L}$ as depicted in Figure 16(a). This may be due to the change in mode shape curvature caused by the

severe damage $\left(87.5 \%\right.$ loss of 'I') overwhelmingly dominating the calculation of $Z_{j}$ and 
distorting the prediction of the light damage (27.1\% loss of ' $\mathrm{I}$ '). The medium damage (65.7\% loss of 'I') was identified as seen in Figure 16(b) because this damage was more severe compared to the light one, as well as the fact that the two damage locations are well separated, providing sufficient changes in mode shape curvature. From Figure 16(b), it was found that locations of both medium and severe damage show different index values, indicating their probability of existence of damage, which reflects well the inflicted damage. This indicates that the DI-P method could possibly be used in the numerical results to indicate the severity of damage, qualitatively.

\subsubsection{Experimental results}

Damage cases discussed in the numerical studies are also reported for the experimental studies to verify the effectiveness of using the damage index method for plate-like structures (DI-P) to detect damage in timber bridges. The single damage cases using the method with the first nine modes are illustrated in Figure 17. For the light damage case g2L (the smallest damage) as depicted in Figure 17(a), the method could not locate the damage at position $(3.375 \mathrm{~m}, 0.9 \mathrm{~m})$. For the medium and severe damage cases g2M and g2S as illustrated in Figures 17(b) and (c), respectively, the damage locations were detected quite clearly even though there are false positives. For single damage cases, it is noticed that the lowest level of damage that could be identified, in the single damage scenarios, is medium damage of case g2M. The light damage for case g2L could not be identified in the experimental studies, which is considered less effective compared to the numerical studies. Nevertheless, the single damage of medium and severe type was identified and this is critical in terms of avoiding catastrophic failure.

The two damage cases considered in the experimental studies using the DI-P method are shown in Figure 18. Observing from the results, severe damage locations at position $(3.375 \mathrm{~m}$, $0.9 \mathrm{~m}$ ) were identified for damage cases g2Sg4L, g2Sg4M and g2Sg4S (see Figures 18(a) to (c)) as well as severe damage at position $(2.25 \mathrm{~m}, 2.1 \mathrm{~m})$ for case $\mathrm{g} 2 \mathrm{Sg} 4 \mathrm{~S}$. However, the light and medium damage at position $(2.25 \mathrm{~m}, 2.1 \mathrm{~m})$ were not identified for cases g2Sg2L and g2Sg2M as depicted in Figures 18(a) and (b), respectively. A possible explanation is that the change in mode shape curvature caused by the severe damage is dominating the results, and overwhelming the curvature change due to the light and medium damage. From the two damage cases, it should be noted that the second damage at position $(2.25 \mathrm{~m}, 2.1 \mathrm{~m})$ was not detected until this damage became roughly comparable in severity to the first severe damage at position $(3.375 \mathrm{~m}, 0.9 \mathrm{~m})$ in the experimental studies. This indicates that the method may encounter problems in identifying multiple damage locations with different degrees of severity using experimental data. However, the method is capable of identifying all severe damage locations and this is critical for ensuring a structure in avoiding catastrophic failure. Detecting medium damage in timber bridges using this method can be achieved as a two stage process. Once the severe damage are identified and repaired, the method can be applied again and then any medium damage can be detected in the absence of any severe damage. Figure 18 (c) of case g2Sg4S shows that both severe damage locations show a relatively similar indication of damage. This indicates that both the severe damage locations have similar probability to be identified as damage. The numerical studies show a better damage detection results compared to the experimental studies. This may be due to the fact that experimental data contains noise and inaccuracies, which can affect the probability of damage being detected.

\section{CONCLUSIONS}


In this paper, finite element (FE) model of a laboratory timber bridge was developed and utilised to investigate, numerically, the robustness of damage detection using the damage index method, extended to plate-like structures incorporating plate theory. The damage index method for platelike structures, denoted as DI-P, was adopted to detect damage in the numerical model of the laboratory timber bridge. From the numerical studies, it was found that using higher number of modes (nine modes) provides better damage detection results compared to just using the first three modes. Hence, the first nine modes were used in the method for all damage cases. From the single and two damage cases, all severe damage was correctly located. The method is also capable of detecting medium damage but not the light damage for two damage cases.

This paper also presents the potential of the DI-P method using experimental data. A laboratory timber bridge was experimentally tested using experimental modal analysis. From the experimental studies, it is clear that the two-dimensional (2-D) interpolation technique (2-D cubic spline) used to expand the limited number of data in the damage detection can significantly affect the detection. Using reconstructed data for detecting damage yields better results than just using 'as is' data. Based on the experimental studies, the method using the first nine modes identified all severe damage in the single and two damage cases. However, the light and medium damage could not be identified except for the single medium damage.

The results of applying the DI-P method to locate damage in the laboratory timber bridge showed that the method was capable of detecting all severe damage for damage cases with less than three damage locations, numerically and experimentally. For medium damage, using the experimental data did not work well in comparison with the use of numerical data. This is mainly due to presence of noise and inaccuracies distorting strain energy changes of actual damage. Despite the good results shown by the method, it is important to acknowledge that due to complexity of real timber bridges, the laboratory model bridge studies may not equal to what is attainable in the field. Real timber bridge testing is planned for future work for validation of this method.

\section{ACKNOWLEDGEMENTS}

The authors wish to thank the Centre for Built Infrastructure Research (CBIR), the School of Civil and Environmental Engineering, University of Technology Sydney for supporting this work. Within the School, the authors would like to thank Dr. Ali Saleh for his advice in finite element modelling using ANSYS. The authors would like to thank Dr. Joko Widjaja and Dr. Christophe Gerber for sharing their opinions and experiences in many aspects of this study. Finally, the authors wish to render thanks to staff of UTS Structures Laboratory for their assistance in conducting the experimental works.

\section{REFERENCES}

1. Department of Transport and Regional Services. 2002-2003 Report on the operation of the Local Government (Financial Assistance) Act 1995. Canberra, Commonwealth of Australia 2003: 74-76.

2. McInnes K. Conserving historic timber bridges. National Trust of Australia (Victoria) 2005: 2.

3. Duwadi SR, Ritter MA, Cesa E. Wood in transportation program: an overview. Fifth International Bridge Engineering Conference, Tampa, Florida, US, National Academy Press, 2000: 310-315.

4. Ross RJ, Brashaw BK, Wang X. Structural condition assessment of in-service wood. Forest Products Journal 2006; 56(6): 4-8.

5. Fridley KJ. Wood Engineering in the 21st century: research needs and goals. Reston, VA, US, American Society of Civil Engineers, 1998.

6. Ou FL, Weller C. An overview of timber bridges. Transportation Research Record 1053, Washington D.C., Transportation Research Board National Research Council, 1986: 1-12. 
7. Li J, Samali B, Crews KI. Determining individual member stiffness of bridge structures using a simple dynamic approach. Acoustics Australia 2004; 32(1): 9-12.

8. Peterson ST, McLean DI, Symans MD, Pollock DG, Cofer WF, Emerson RN, Fridley KJ. Application of dynamic system identification to timber beams I. Journal of Structural Engineering 2001; 127(4): 418-425.

9. Peterson ST, McLean DI, Symans MD, Pollock DG, Cofer WF, Emerson RN, Fridley KJ. Application of dynamic system identification to timber beams II. Journal of Structural Engineering 2001; 127(4): 426-432.

10. Peterson ST, McLean DI, Pollock DG. Application of dynamic system identification to timber bridges. Journal of Structural Engineering 2003; 129(1): 116-124.

11. Yang XY, Ishimaru Y, Iida I, Urakami H. Application of modal analysis by transfer function to nondestructive testing of wood I: determination of localized defects in wood by the shape of flexural vibration wave. Journal of Wood Science 2002; 48: 283-288.

12. Morison A, VanKarsen CD, Evensen HA, Ligon JB, Erickson JR, Ross RJ, Forsman JW. Timber bridge evaluation: a global nondestructive approach using impact generated FRFs. Proceedings of IMAC-XX, Los Angeles, California, US, Society for Experimental Mechanics Inc., 2002: 1567-1573.

13. Morison A, VanKarsen CD, Evensen HA, Ligon JB, Erickson JR, Ross RJ, Forsman JW. Dynamic characteristics of timber bridges as a measure of structural integrity. Proceedings of IMAC-XXI, Kissimmee, Florida, US, Society for Experimental Mechanics Inc., 2003: (Published on CD-ROM).

14. Hu YC, Nakao T, Nakai T, Gu J, Wang F. Dynamic properties of three types of wood-based composites. Journal of Wood Science 2005; 51: 7-12.

15. Hu YC, Nakao T, Nakai T, Gu J, Wang F. Vibration properties of wood plastic plywood. Journal of Wood Science 2005; 51: 13-17.

16. Hu C, Afzal MT. A statistical algorithm for comparing mode shapes of vibration testing before and after damage in timbers. Journal of Wood Science 2006; 52: 348-352.

17. Hu C, Afzal MT. A wavelet analysis-based approach for damage localization in wood beams. Journal of Wood Science 2006; 52: 456-460.

18. Ross RJ, Brashaw BK, Wang, X. Structural condition assessment of in-service wood. Forest Products Journal 2006; 56(6): 4-8.

19. Bale S, Browne CA. Non-destructive identification of defects in timber bridges. Road System and Engineering Technology Forum, The Bardon Centre, Brisbane, Department of Main Roads, Queensland Government, 2004.

20. Wilkinson K, Thambiratnam D, Ferreira L. Non destructive testing of timber bridge girders. International Conference on Structural Condition Assessment, Monitoring and Improvement, Perth, Western Australia, 2005: (Published on CD-ROM).

21. ANSYS release 10.0 documentation, ANSYS Inc., Canonburg, US, 2005.

22. Samali B, Choi FC, Li J, Crews K. Experimental investigations on a laboratory timber bridge using damage index method for plate-like structures. 5th Australasian Congress on Applied Mechanics, Brisbane, Australia, 10th-12th December 2007: 114-119.

23. LMS CADA-X modal analysis manual, LMS International, Belgium, 1992.

24. Cornwell P, Doebling SW, Farrar CR. Application on the strain energy damage detection method to plate-like structures. Journal of Sound and Vibration 1999; 224(2): 359-374.

25. Park S, Stubbs N. Reconstruction of mode shapes using Shannon's sampling theorem and its application to the nondestructive damage localization algorithm. Smart Structures and Materials, Smart Systems for Bridges, Structures, and Highways, San Diego, California, US, SPIE- The International Society for Optical Engineering, 1995: 280-292.

26. Park S, Stubbs N. Bridge diagnostics via vibration monitoring. Smart Structures and Materials, Smart Systems for Bridges, Structures, and Highways, edited by Matthews LK, SPIE- The International Society for Optical Engineering, San Diego, California, US, 1996; 2719: 36-45.

27. Wang T-L, Zong Z. Improvement of evaluation method for existing highway bridges. Research Report No. FL/DOT/RMC/6672-818, Department of Civil and Environmental Engineering, Florida International University, Tallahassee, Florida, 2002.

28. Pereyra LR, Osegueda RA, Carrasco CJ, Ferregut CM. Damage detection in a stiffened plate using modal strain energy differences. Proceedings of SPIE- Nondestructive Evaluation of Aging Aircraft, Airports, and Aerospace Hardware III, edited by Mal AK, SPIE- The International Society for Optical Engineering, Newport Beach, California, US, 1999; 3586, DOI: 10.1117/12.339887.

29. Worden K, Manson G, Allman DJ. An experimental appraisal of the strain energy damage location method. Proceedings of the 4th International Conference on Damage Assessment of Structures, edited by Holford KM, 
Brandon JA, Dulieu-Barton JM, Gilchrist MD, Worden K, Trans Tech Publications Ltd, Cardiff, Wales, UK, 2001: 35-46.

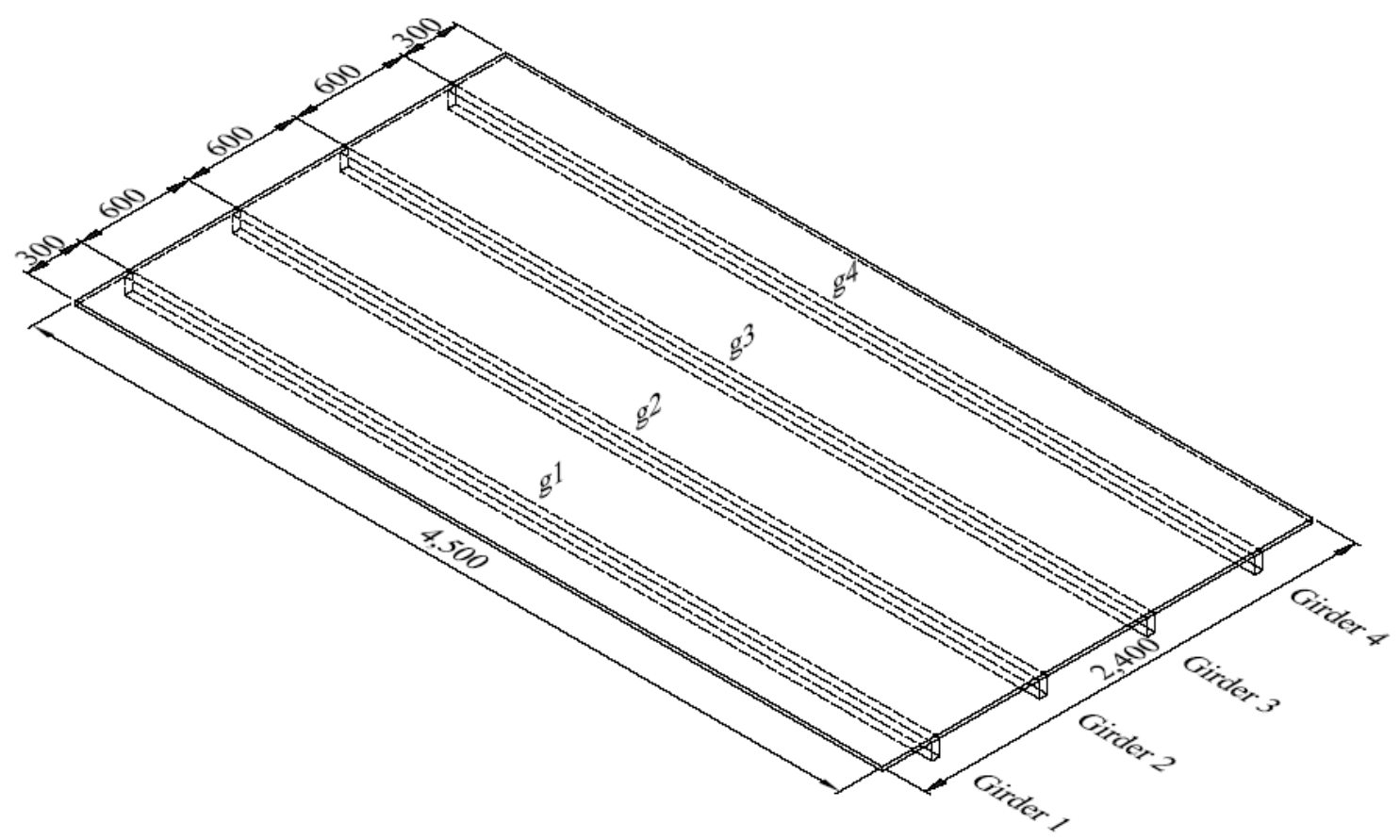

Figure 1. Geometric configuration of the laboratory timber bridge. 


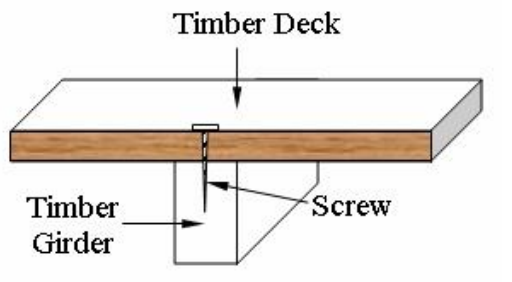

(a) Cross section.

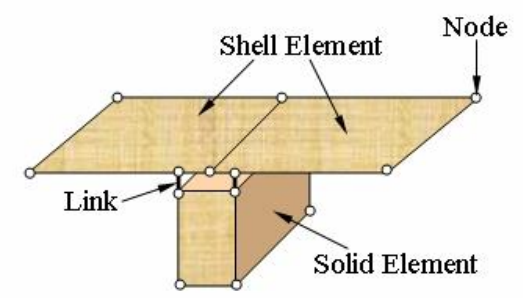

(b) Finite element.

Figure 2. Girder-deck connection. 


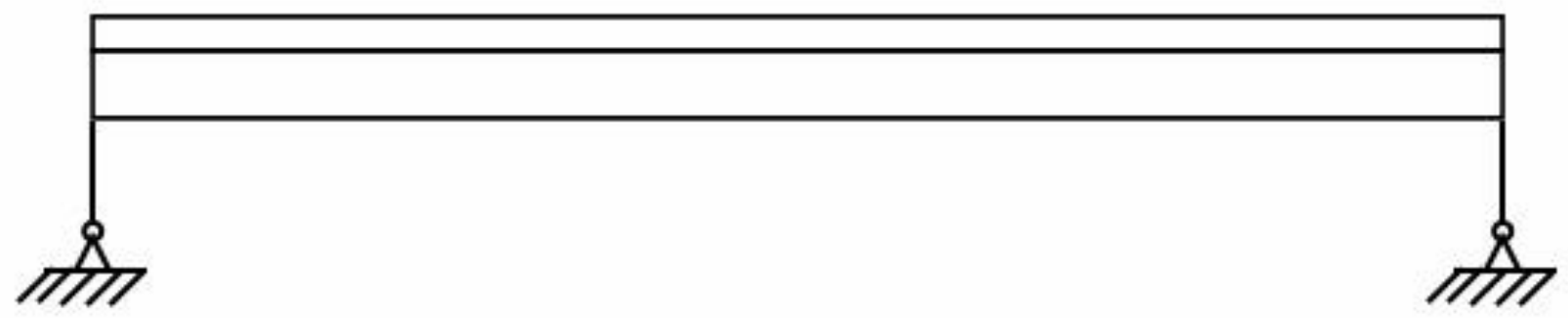

Figure 3. Modelling of bridge supports. 


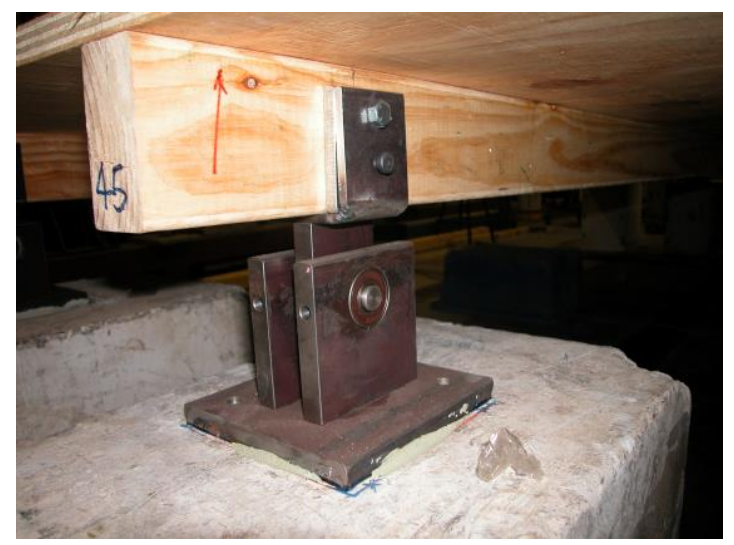

Figure 4. Fabricated pin support. 


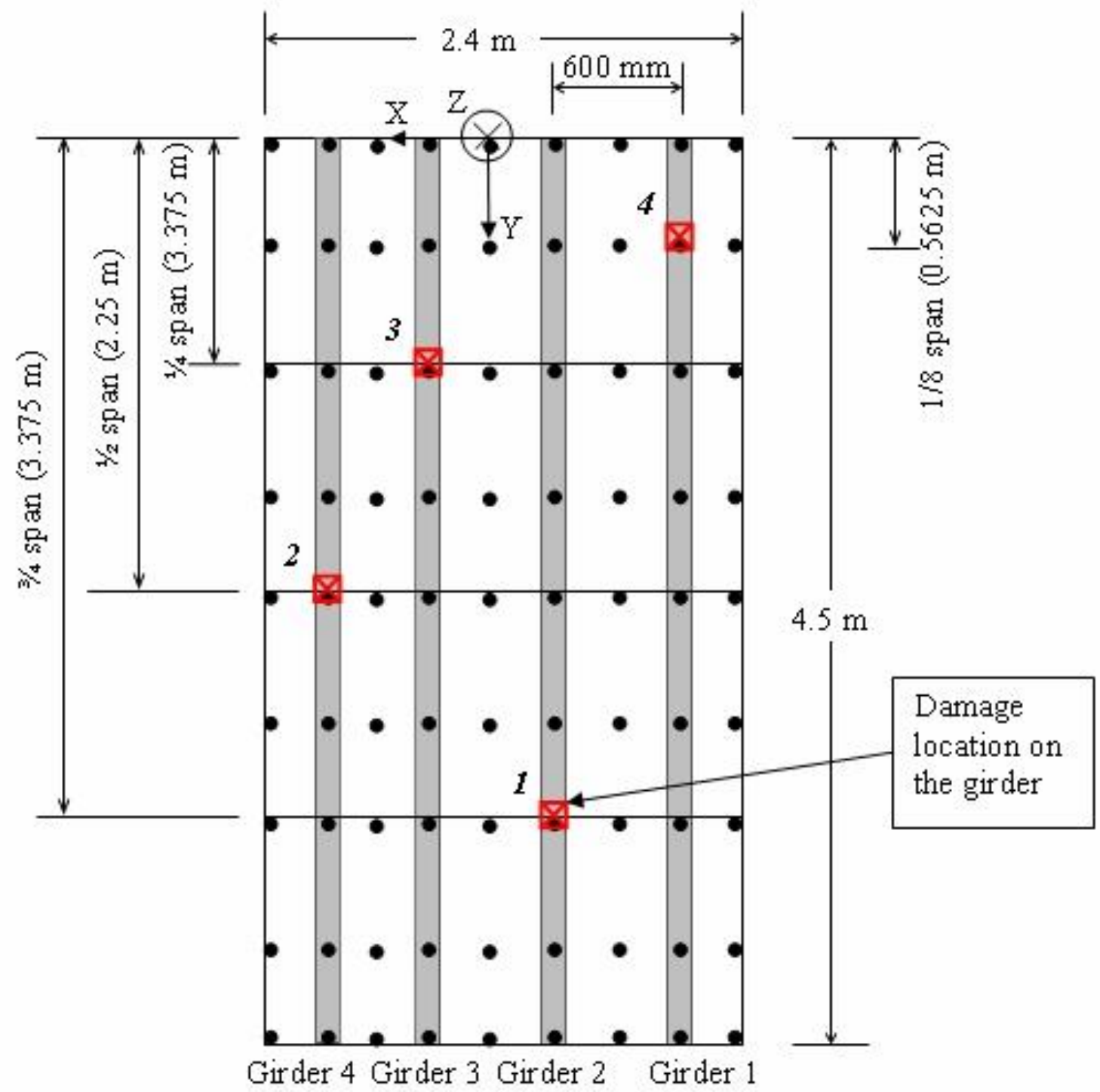

Figure 5. Plan view of damage locations on the bridge model. 


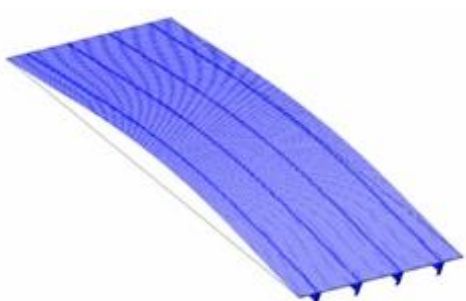

$(8.94 \mathrm{~Hz})$

Mode 1 (1st flexural)

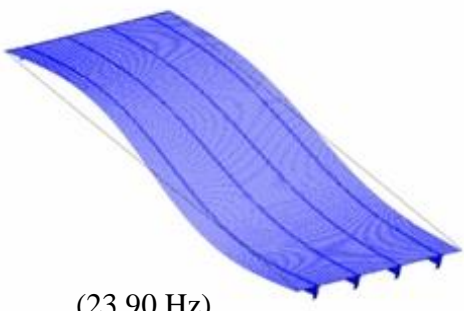

$(23.90 \mathrm{~Hz})$

Mode 4 (2nd flexural)

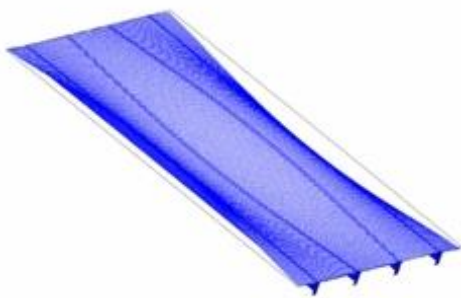

$(40.04 \mathrm{~Hz})$

Mode 7 (2nd transversal)

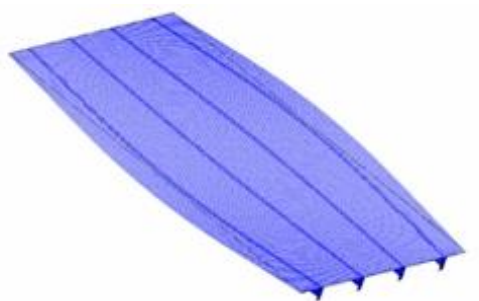

$(9.64 \mathrm{~Hz})$

Mode 2 (1st torsional)

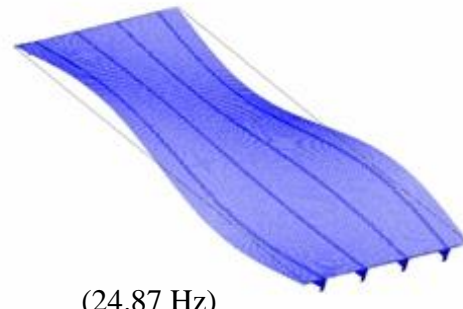

$(24.87 \mathrm{~Hz})$

Mode 5 (2nd torsional)

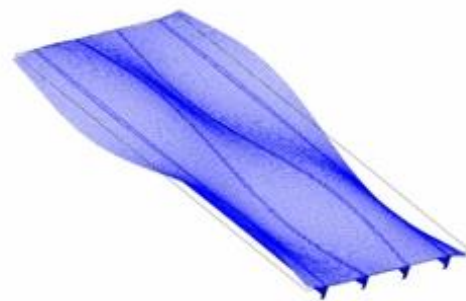

$(47.13 \mathrm{~Hz})$

Mode 8 (2nd transversal \& $2^{\text {nd }}$ flexural)

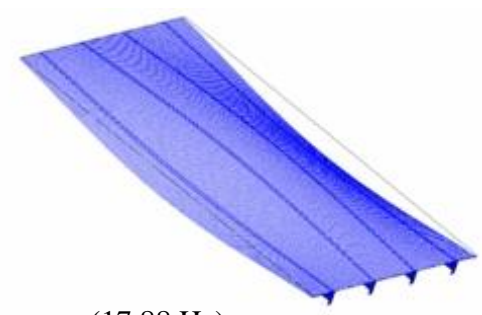

$(17.88 \mathrm{~Hz})$

Mode 3 (1st transversal)

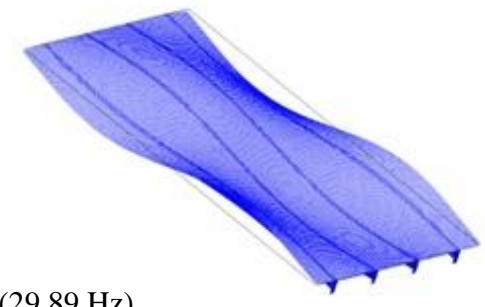

$(29.89 \mathrm{~Hz})$

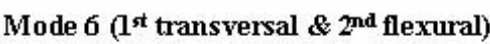

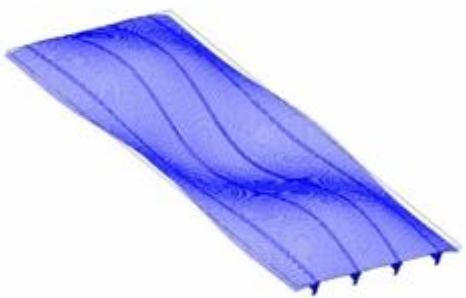

$(53.72 \mathrm{~Hz})$

Mode 9 ( ${ }^{\text {rd }}$ flexural)

Figure 6. Nine vibration mode shapes of the laboratory timber bridge FE model. 


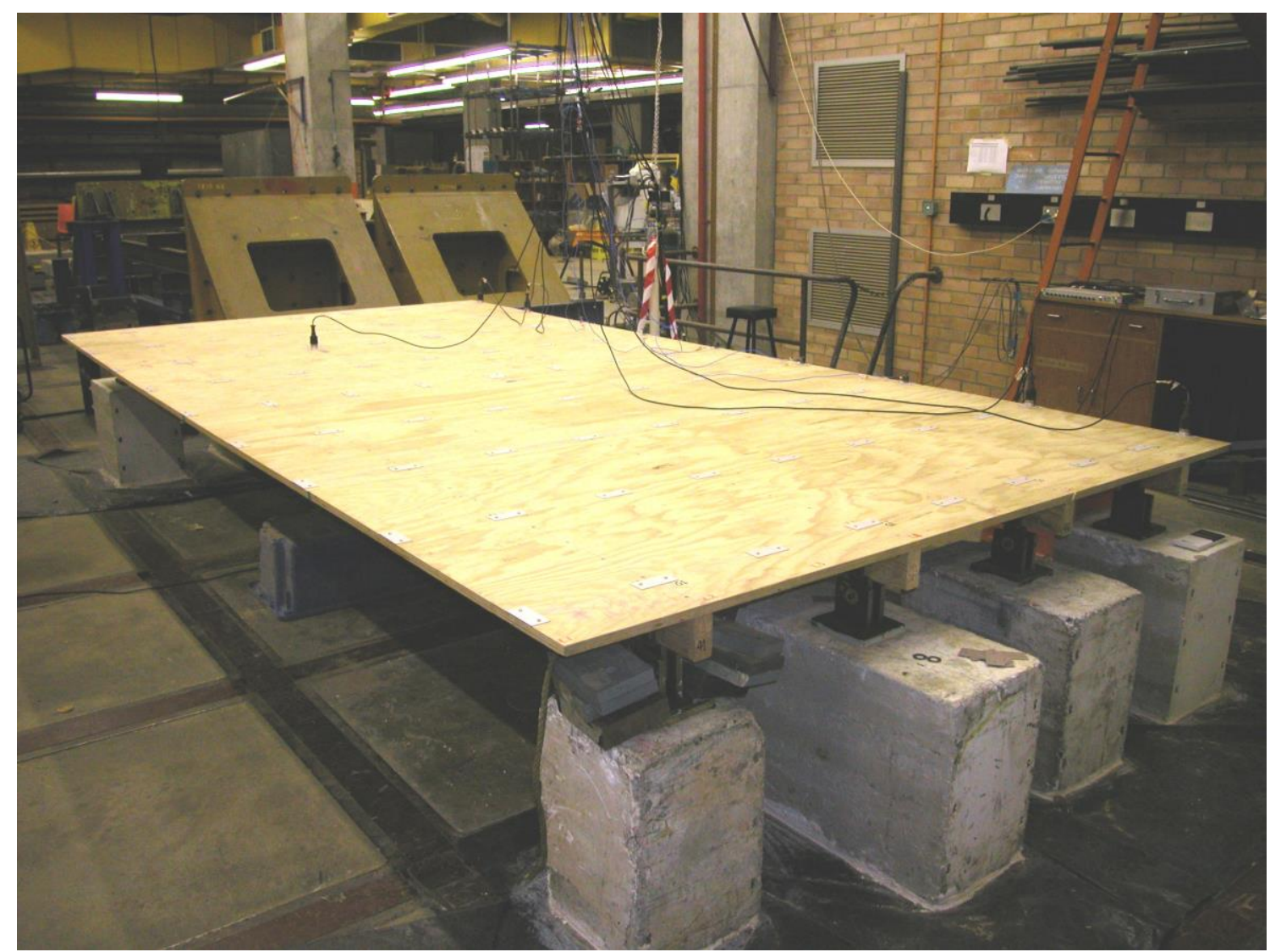

Figure 7. The laboratory timber bridge. 


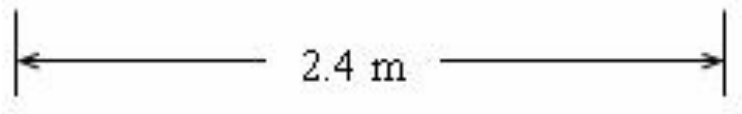

L9 $\begin{array}{llllllll} & \text { L7 } & \text { L6 } & \text { L5 } & \text { L4 } & \text { L3 } & \text { L2 } & \text { L1 }\end{array}$

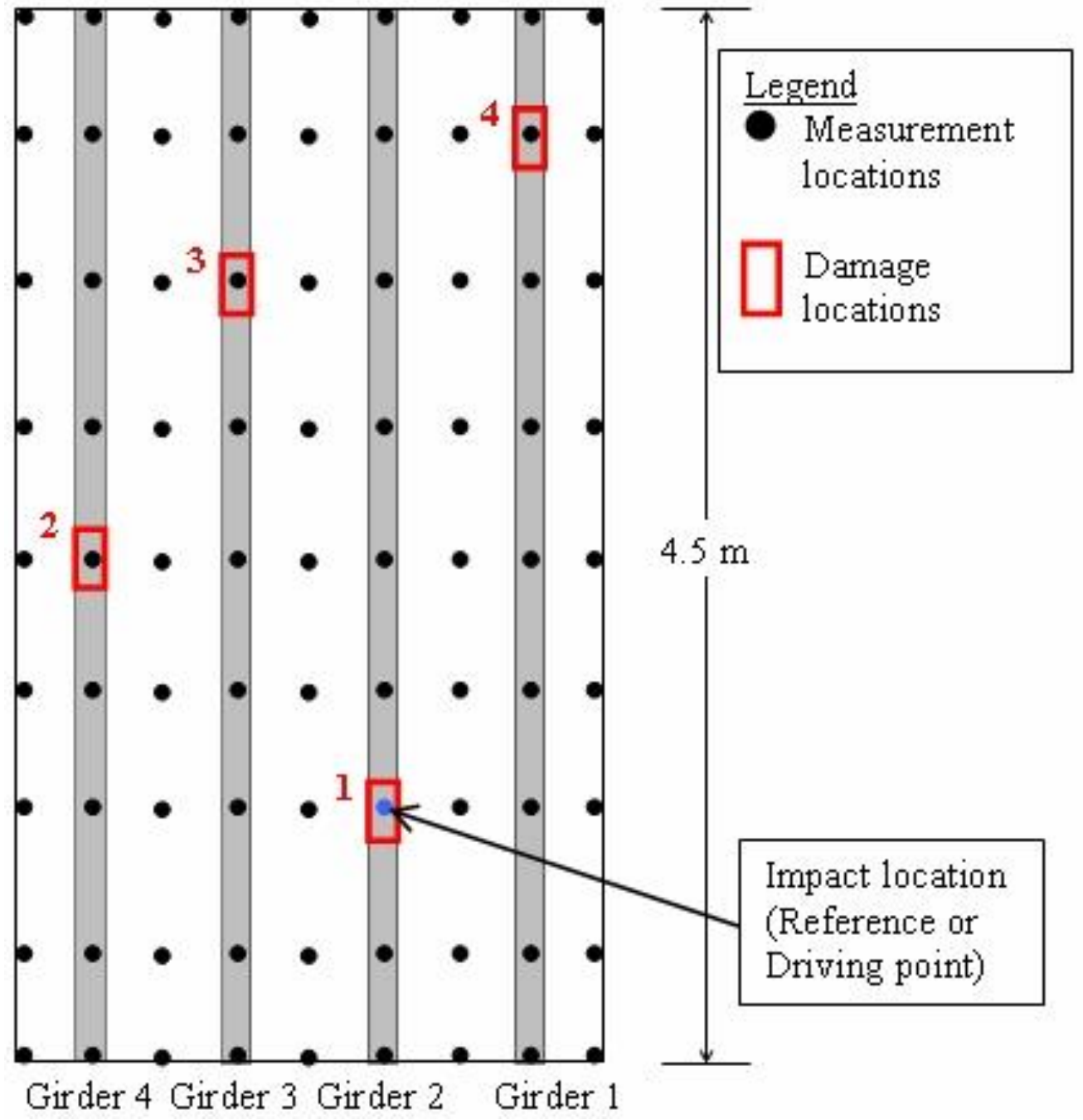

Figure 8. Plan view of damage locations and measurement locations on the bridge. 


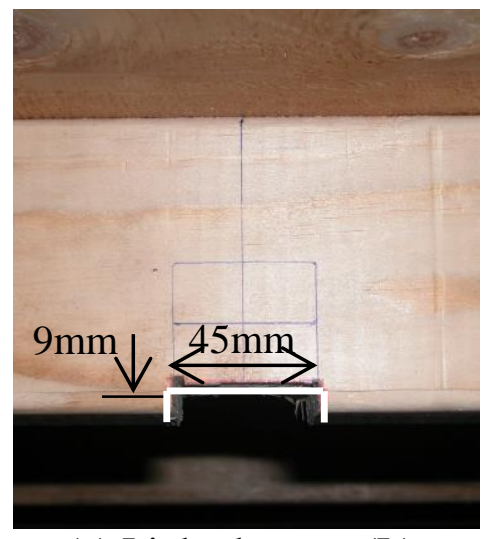

(a) Light damage (L).

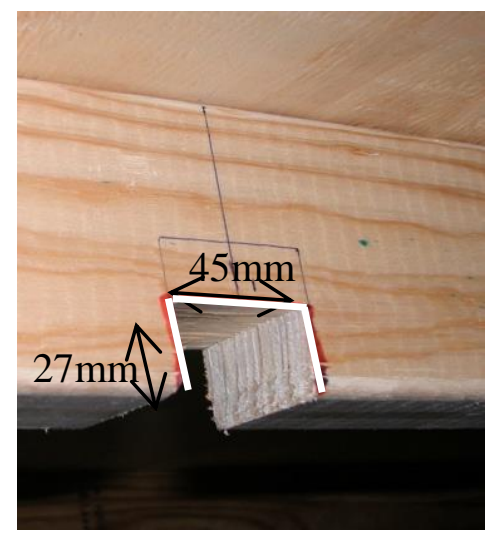

(b) Medium damage (M).

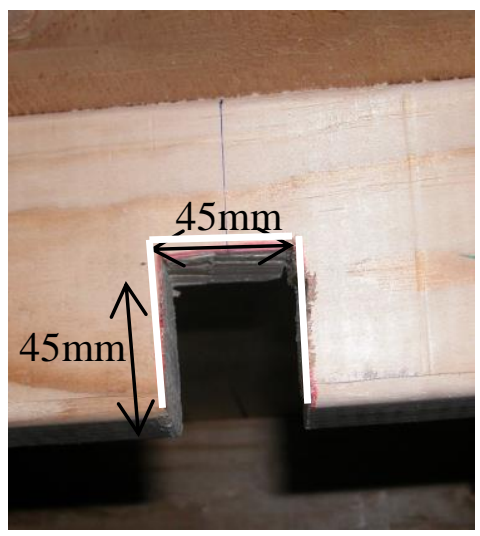

(c) Severe damage (S). Figure 9. Side view of various inflicted damage. 


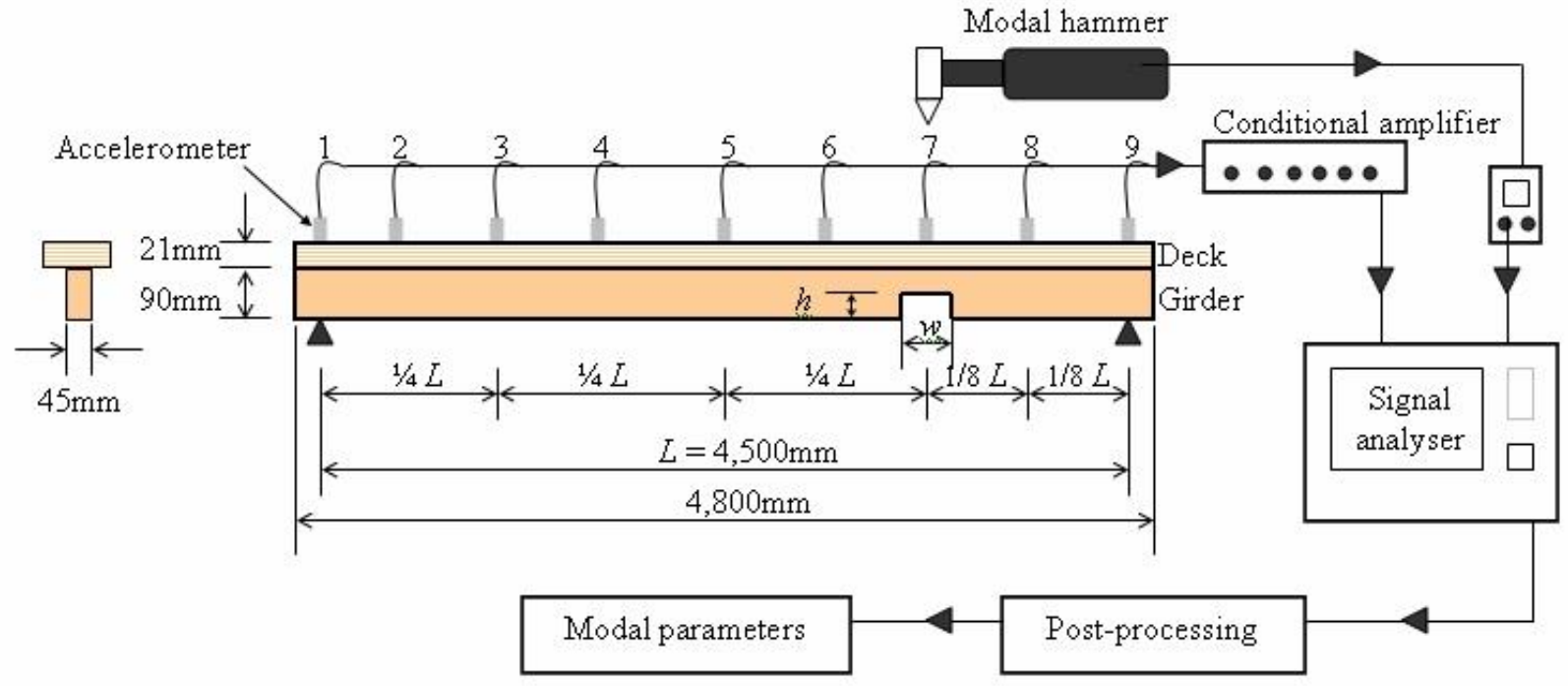

Figure 10. Modal tests on the laboratory timber bridge. 


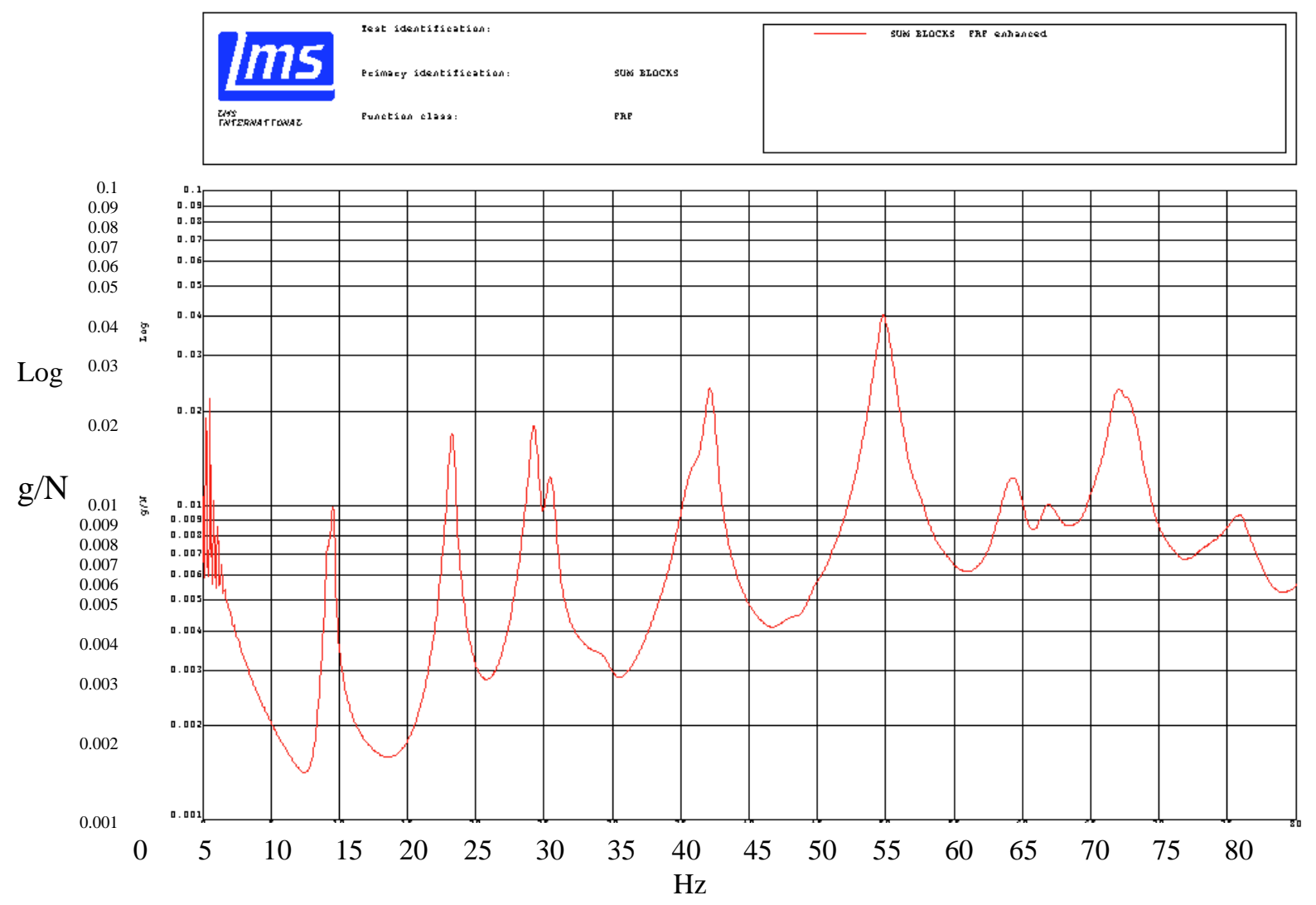

Figure 11. A typical FRF spectrum for the laboratory timber bridge. 


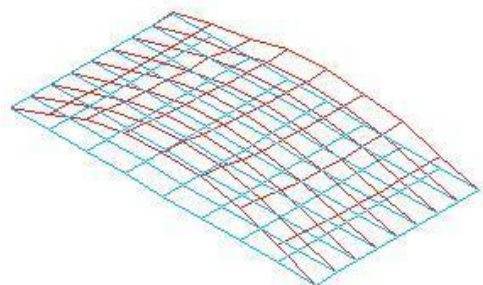

mode $1\left(1^{\text {st }}\right.$ flexural $)$ $(9.06 \mathrm{~Hz})$

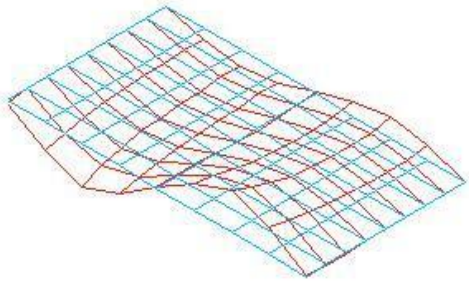

mode 4 ( $2^{\text {nd }}$ flexural)

$(24.17 \mathrm{~Hz})$

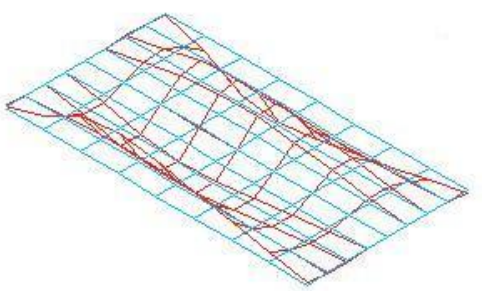

mode 7 ( $2^{\text {nd }}$ transversal $)$ $(37.06 \mathrm{~Hz})$

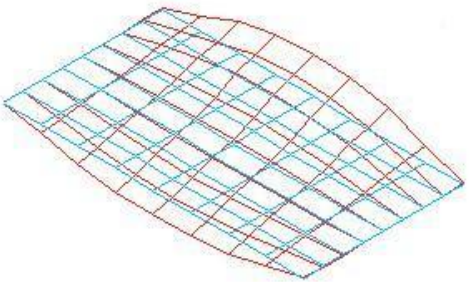

mode 2 ( $1^{\text {st }}$ torsional $)$

$(9.49 \mathrm{~Hz})$

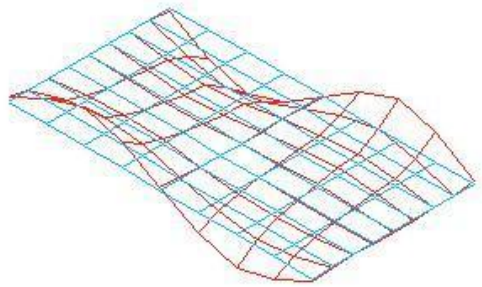

mode 5 ( $2^{\text {nd }}$ torsional $)$

$(25.32 \mathrm{~Hz})$

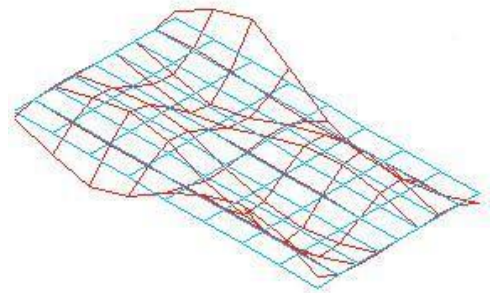

mode $8\left(2^{\text {nd }}\right.$ transversal $\& 2^{\text {nd }}$ flexural)

$(49.71 \mathrm{~Hz})$

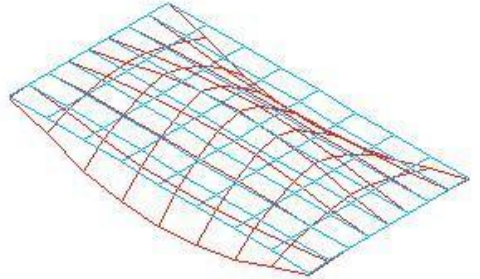

mode $3\left(1^{\text {st }}\right.$ transversal $)$ $(18.07 \mathrm{~Hz})$

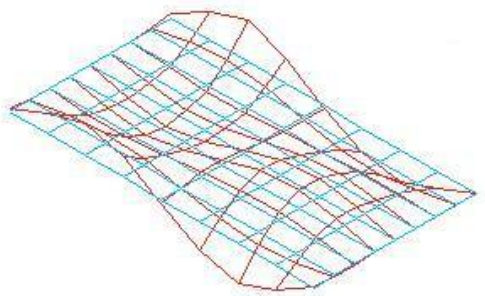

mode $6\left(1^{\text {st }}\right.$ transversal $\& 2^{\text {nd }}$ flexural) $(35.45 \mathrm{~Hz})$

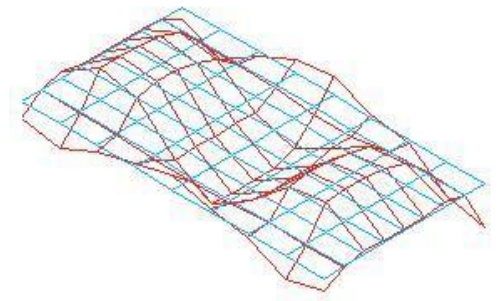

mode 9 ( $3^{\text {rd }}$ flexural $)$ $(59.25 \mathrm{~Hz})$

Figure 12. Experimental mode shapes (81-points) for the laboratory timber bridge. 


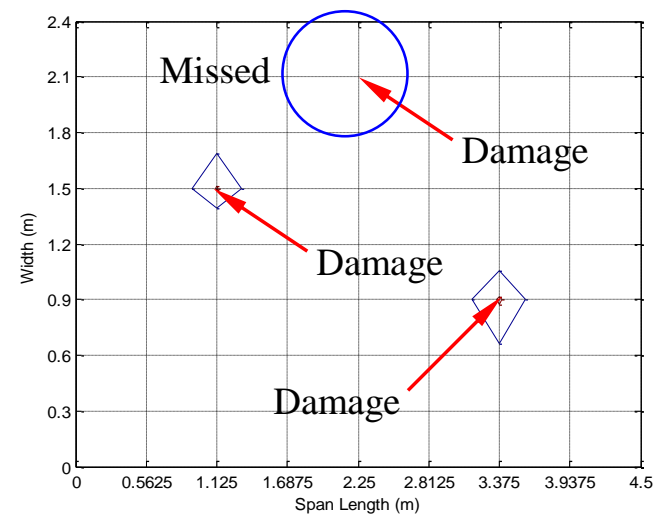

(a) Damage localisation results using "as is" data (9 modes).

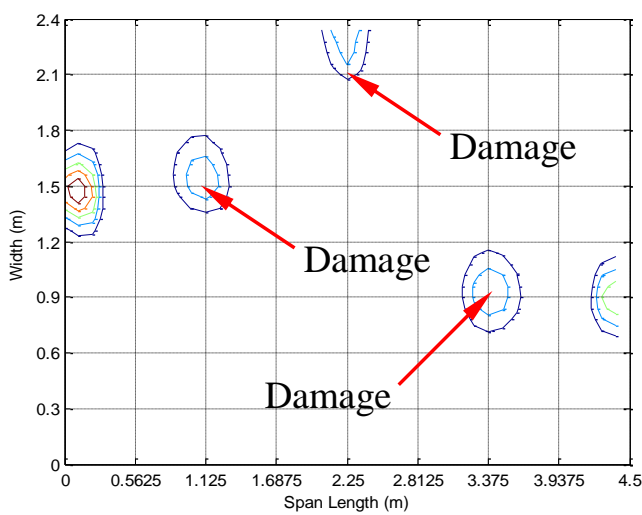

(b) Damage localisation results using 2-D cubic spline interpolation (9 modes).

Figure 13. A three damage scenario case g2Sg4Sg3S using the experimental data. 

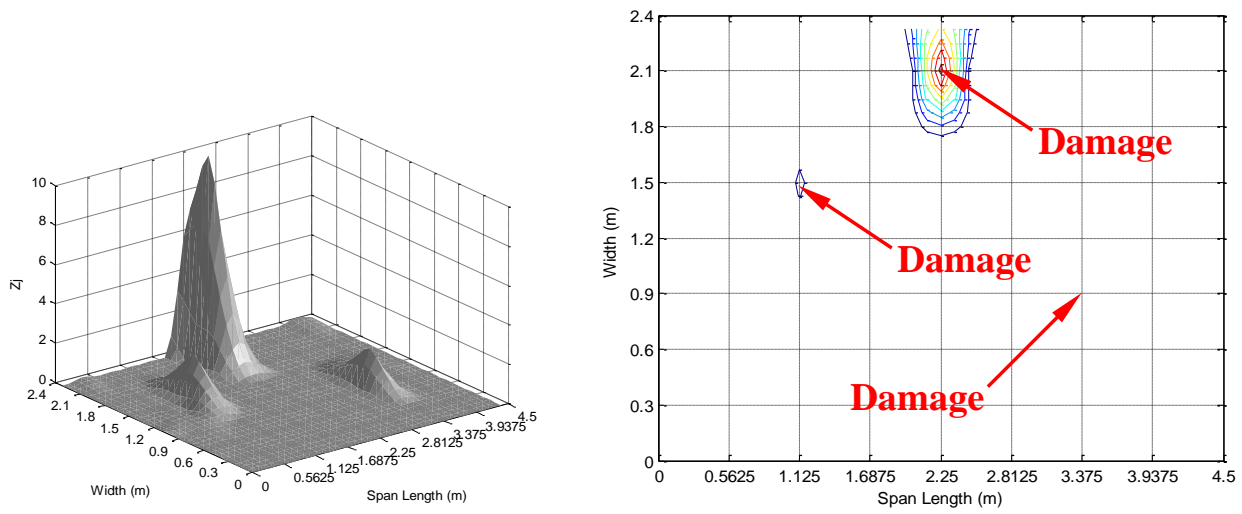

(a) Damage case g2 $\mathrm{Sg} 4 \mathrm{Sg} 3 \mathrm{~S}$ using 3 modes.
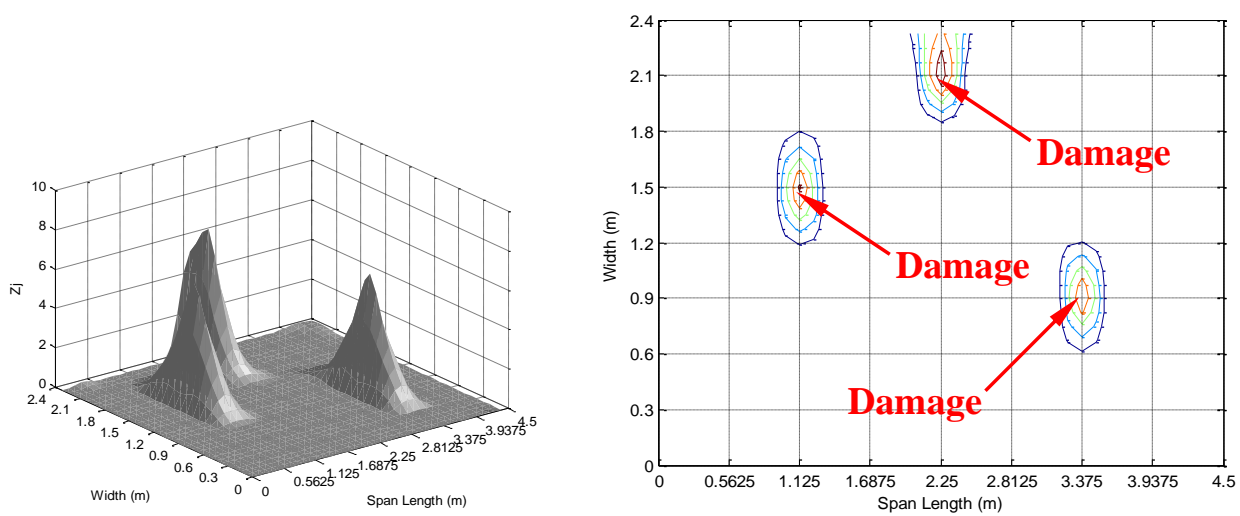

(b) Damage case g2Sg4Sg3S using 9 modes.

Figure 14. A three damage case $\mathrm{g} 2 \mathrm{Sg} 4 \mathrm{Sg} 3 \mathrm{~S}$ using the numerical data. 


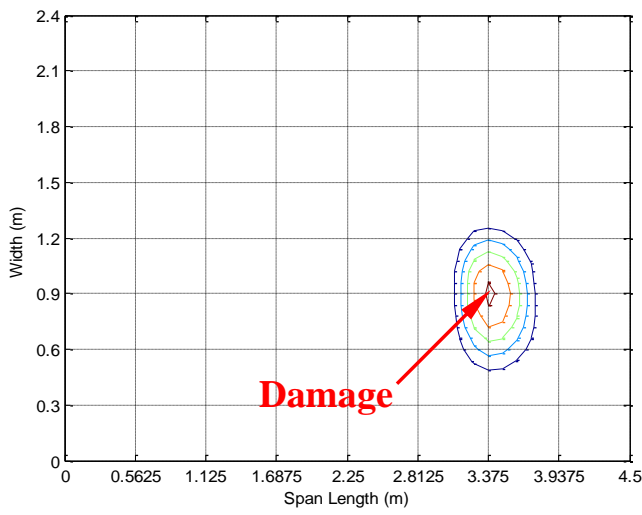

(a) g2L (light damage).

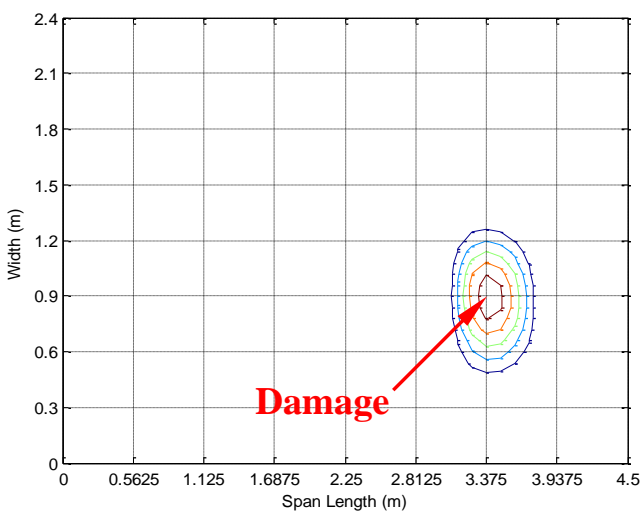

(b) g2M (medium damage).

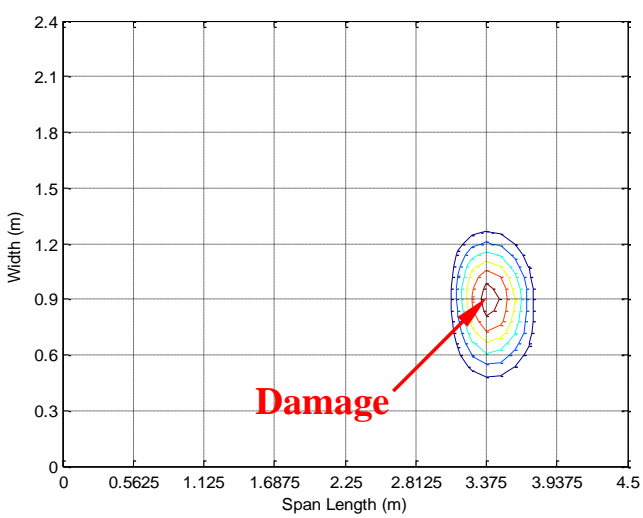

(c) g2S (severe damage).

Figure 15. Single damage cases using the numerical data. 


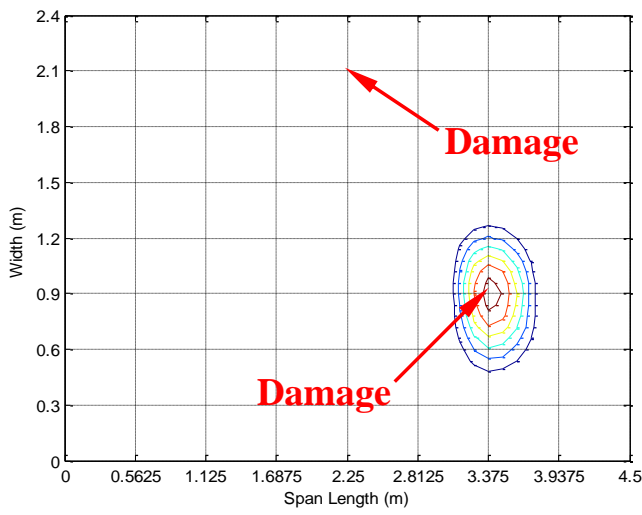

(a) g2Sg4L.

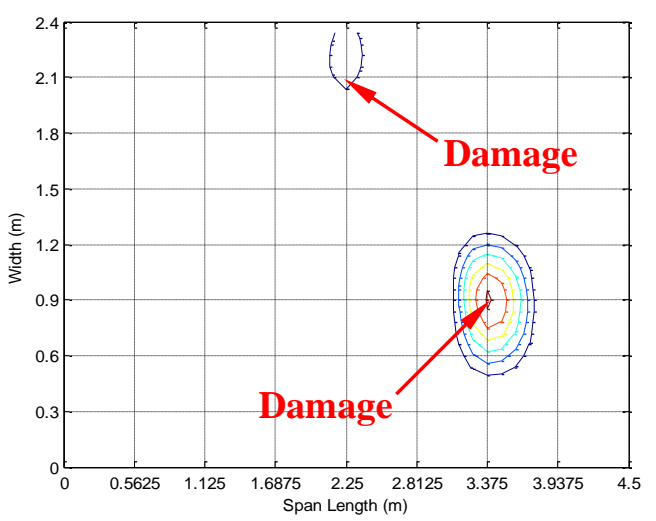

(b) g2Sg4M.

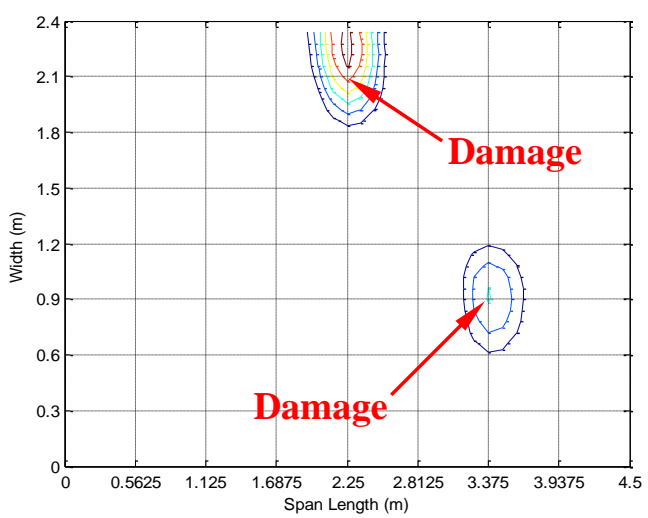

(c) g2Sg4S.

Figure 16. Two damage cases using the numerical data. 


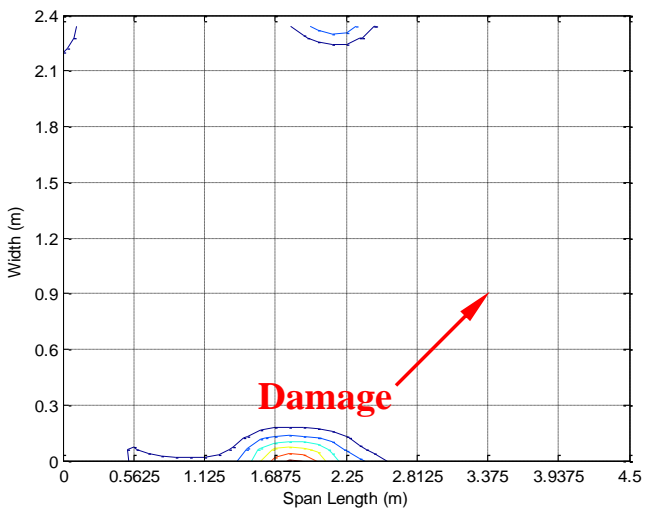

(a) g2L.

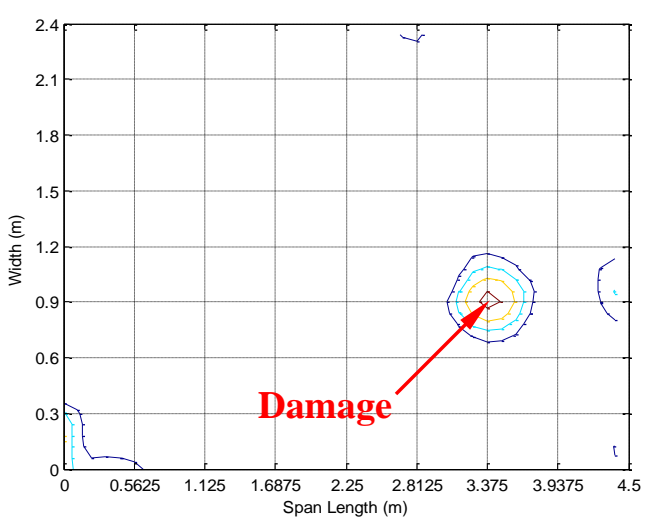

(b) g2M.

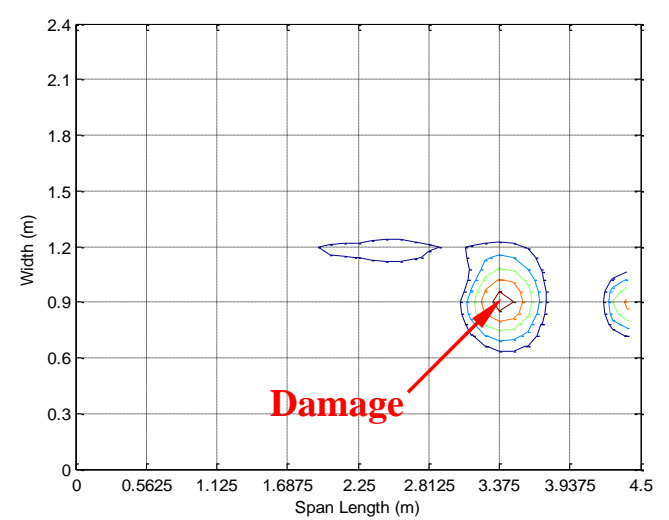

(c) g2S.

Figure 17. Single damage cases using the experimental data. 


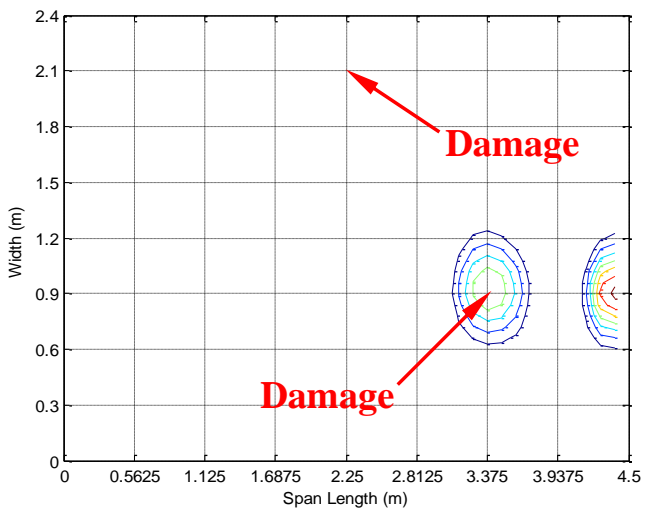

(a) $\mathrm{g} 2 \mathrm{Sg} 4 \mathrm{~L}$.

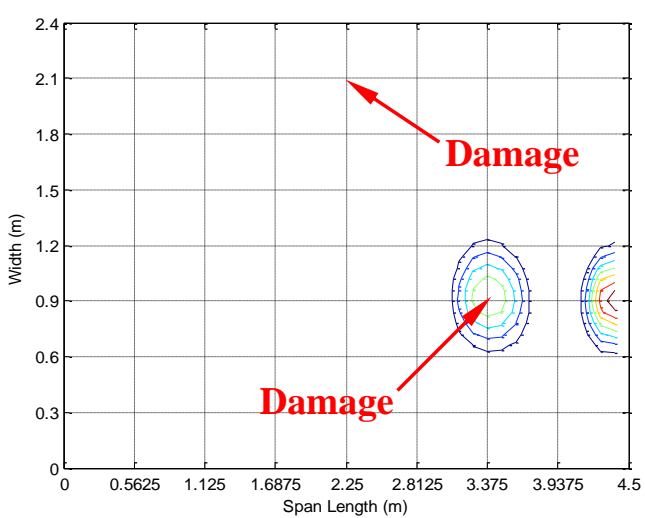

(b) $\mathrm{g} 2 \mathrm{Sg} 4 \mathrm{M}$.

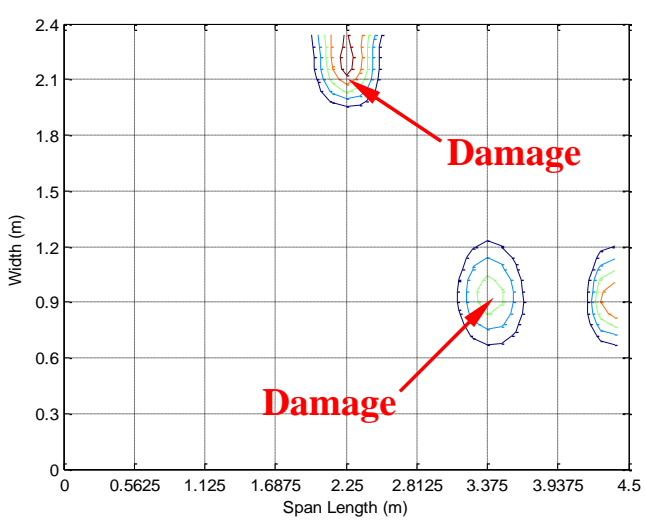

(c) $\mathrm{g} 2 \mathrm{Sg} 4 \mathrm{~S}$.

Figure 18. Two damage cases using the experimental data. 
Table 1. Damage scenarios for the analytical model of the laboratory timber bridge.

\begin{tabular}{|l|l|l|l|l|l|}
\hline $\begin{array}{l}\text { Damage } \\
\text { Case }\end{array}$ & $\begin{array}{l}\text { Damage } \\
\text { Scenario }\end{array}$ & $\begin{array}{l}\text { Location per 8th of } \\
\text { span length/Girder } \\
\text { number }\end{array}$ & $\begin{array}{l}\text { Length } \\
l \\
(\mathrm{~mm})\end{array}$ & $\begin{array}{l}\text { Depth } \\
h \\
(\mathrm{~mm})\end{array}$ & \% loss of 'I' \\
\hline Deck 2 & $\mathrm{D} 2$ & Undamaged & - & - & - \\
\hline 1 & $\mathrm{~g} 2 \mathrm{~L}$ & $6 / \mathrm{g} 2$ & 45 & 9 & 27.1 \\
\hline 2 & $\mathrm{~g} 2 \mathrm{M}$ & $6 / \mathrm{g} 2$ & 45 & 27 & 65.7 \\
\hline 3 & $\mathrm{~g} 2 \mathrm{~S}$ & $6 / \mathrm{g} 2$ & 45 & 45 & 87.5 \\
\hline 4 & $\mathrm{~g} 2 \mathrm{Sg} 4 \mathrm{~L}$ & $6 / \mathrm{g} 2,4 / \mathrm{g} 4$ & 45 & 45,9 & $87.5,27.1$ \\
\hline 5 & $\mathrm{~g} 2 \mathrm{Sg} 4 \mathrm{M}$ & $6 / \mathrm{g} 2,4 / \mathrm{g} 4$ & 45 & 45,27 & $87.5,65.7$ \\
\hline 6 & $\mathrm{~g} 2 \mathrm{Sg} 4 \mathrm{~S}$ & $6 / \mathrm{g} 2,4 / \mathrm{g} 4$ & 45 & All 45 & All 87.5 \\
\hline 7 & $\mathrm{~g} 2 \mathrm{Sg} 4 \mathrm{Sg} 3 \mathrm{~L}$ & $6 / \mathrm{g} 2,4 / \mathrm{g} 4,2 / \mathrm{g} 3$ & 45 & $45,45,9$ & $87.5,87.5,27.1$ \\
\hline 8 & $\mathrm{~g} 2 \mathrm{Sg} 4 \mathrm{Sg} 3 \mathrm{M}$ & $6 / \mathrm{g} 2,4 / \mathrm{g} 4,2 / \mathrm{g} 3$ & 45 & $45,45,27$ & $87.5,87.5,65.7$ \\
\hline 9 & $\mathrm{~g} 2 \mathrm{Sg} 4 \mathrm{Sg} 3 \mathrm{~S}$ & $6 / \mathrm{g} 2,4 / \mathrm{g} 4,2 / \mathrm{g} 3$ & 45 & All 45 & All 87.5 \\
\hline 10 & $\mathrm{~g} 2 \mathrm{Sg} 4 \mathrm{Sg} 3 \mathrm{Sg} 1 \mathrm{~L}$ & $6 / \mathrm{g} 2,4 / \mathrm{g} 4,2 / \mathrm{g} 3,1 / \mathrm{g} 1$ & 45 & $45,45,45,9$ & $87.5,87.5,87.5,27.1$ \\
\hline 11 & $\mathrm{~g} 2 \mathrm{Sg} 4 \mathrm{Sg} 3 \mathrm{Sg} 1 \mathrm{M}$ & $6 / \mathrm{g} 2,4 / \mathrm{g} 4,2 / \mathrm{g} 3,1 / \mathrm{g} 1$ & 45 & $45,45,45,27$ & $87.5,87.5,87.5,65.7$ \\
\hline 12 & $\mathrm{~g} 2 \mathrm{Sg} 4 \mathrm{Sg} 3 \mathrm{Sg} 1 \mathrm{~S}$ & $6 / \mathrm{g} 2,4 / \mathrm{g} 4,2 / \mathrm{g} 3,1 / \mathrm{g} 1$ & 45 & All 45 & All 87.5 \\
\hline
\end{tabular}

(e.g. g2Sg4Sg3M denotes a cumulative damage scenario of medium damage on $1 / 4$ span $(1.125 \mathrm{~m})$ of girder 3 , severe damage on $3 / 4$ span $(3.375 \mathrm{~m})$ of girder 2 and $1 / 2$ span $(2.25 \mathrm{~m})$ of girder 4) 